\title{
HOLOMORPHIC MAPS INTO COMPLEX PROJECTIVE SPACE OMITTING HYPERPLANES $\left({ }^{1}\right)$
}

\author{
BY
}

MARK L. GREEN

\begin{abstract}
Using methods akin to those of E. Borel and R. Nevanlinna, a generalization of Picard's theorem to several variables is proved by reduction to a lemma on linear relations among exponentials of entire functions. More specifically, it is shown that a holomorphic map from $C^{m}$ to $\mathbf{P}_{n}$ omitting $n+2$ distinct hyperplanes has image lying in a hyperplane. If the map omits $n+2$ or more hyperplanes in general position, the image will lie in a linear subspace of low dimension, being forced to be constant if the map omits $2 n+1$ hyperplanes in general position. The limits found for the dimension of the image are shown to be sharp.
\end{abstract}

Introduction. We will establish two generalizations of Picard's theorem:

Theorem 1. Let f: $\mathbf{C}^{m} \rightarrow \mathbf{P}_{n}$ be a bolomorphic map that omits $n+k$ byperplanes in general position, $k \geq 1$. Then the image of $f$ is contained in a projective linear subspace of dimension $\leq[n / k]$, where the brackets mean greatest integer. Furthermore, this bound is sharp.

Corollary 1. A bolomorpbic map $f: \mathbf{C}^{m} \rightarrow \mathbf{P}_{n}$ that misses $n+2$ byperplanes in general postion has image contained in a linear subspace of dimension [ $n / 2]$, bence in a proper linear subspace.

Corollary 2. A bolomorphic map $f: \mathbf{C}^{m} \rightarrow \mathbf{P}_{n}$ that misses $2 n+1$ byperplanes in general position is constant.

These corollaries resolve conjectures of S. S. Chern [3] and H. Wu ([7], [8]). The dimension $[n / 2]$ in Corollary 1 improves Chern's original conjecture. A weaker bound on the dimension of the linear subspace generated by the image will be obtained when the hypothesis of general position is dropped, as follows:

Theorem 2. A bolomorpbic map $f: \mathbf{C}^{m} \rightarrow \mathbf{P}_{n}$ that omits any distinct $n+2$ byperplanes bas image lying in a proper projective linear subspace.

Received by the editors October 18, 1971.

AMS 1970 subject classifications. Primary 30A70, 32H25; Secondary 32H20.

Key words and phrases. Picard theorem, value distribution theory, exponential function, Nevanlinna theory, hyperplanes in general position, holomorphic curve, Nevanlinna characteristic function.

(1)This research was done while the author held an NSF Graduate Fellowship and a Procter Fellowship at Princeton University. 
The proofs are entirely analytical, inspired by the approach to the subject exemplified by R. Nevanlinna's Le théorème de Picard-Borel et la théorie des fonctions méromorphes, and will be parallel to Emile Borel's proof of Picard's theorem in 1897 [1].

Before embarking on the full proofs, we will give a skeleton key to what follows by a brief discussion of the case of $n+2$ hyperplanes in general position in $\mathbf{P}_{n}$. Using homogeneous coordinates $z_{0}, \cdots, z_{n}$, the hyperplanes may be assumed to be $\left\{z_{i}=0\right\}, i=0, \cdots, n$, and $\left\{z_{0}+\cdots+z_{n}=0\right\}$. Writing the map $f$ in global homogeneous coordinates as $\left(f_{0}, \cdots, f_{n}\right)$, since the $f_{i}$ are without poles or zeros, they may be expressed as exponentials $\exp \left(g_{0}\right), \ldots$, exp $\left(g_{n}\right)$, where the $g_{i}$ : $\mathrm{C}^{m} \rightarrow \mathrm{C}$ are holomorphic. Further, because their sum is never zero, as $f$ omits $\left\{z_{0}+\cdots+z_{n}=0\right\}$ and has nopoles, it likewise is an exponential $\exp \left(g_{n+1}\right)$ satisfying the equation

$$
\exp \left(g_{0}\right)+\cdots+\exp \left(g_{n}\right)+\exp \left(i \pi+g_{n+1}\right) \equiv 0 .
$$

What must be done is to show that this equation is absurd unless $\forall i \exists_{j}$ such that $g_{i}-g_{j}$ is constant, $i \neq j$, in which case $f_{i} / f_{j}$ is constant and hence $f$ goes into a proper linear subspace. When the domain is just the complex line, and indeed a reduction to this case will be possible, this is a classical result of the defect relations in the theory of holomorphic curves (see, for example, Proposition 5.15 of [7]). However, the result is accessible directly and will be proved by fairly elementary means. The essential idea, in order to show the impossibility of a linear relation among "independent" exponentials of holomorphic functions (independent means not differing by a constant), is to find some way to utilize the crucial fact about an exponential- the differential equation it satisfies. Fortunately, it is only necessary to do this in a crude way, namely we divide through the equation by the last term getting

$$
\exp \left(g_{0}-g_{n+1}\right)+\cdots+\exp \left(g_{n}-g_{n+1}\right) \equiv 1 \text {. }
$$

Then we differentiate (assuming the domain of the $g_{i}$ is the complex line) obtaining an equation of shorter length, but where the coefficients of the exponentials are nonconstant holomorphic functions. An induction is still possible providing there is a method of keeping track of the fact that the coefficients of the equation of exponentials have "rates of growth" small with respect to the exponentials themselves. Since in later steps the coefficients will involve quotients of derivatives of the $g_{i}$, they will be meromorphic functions, so that the maximum modulus will not serve our purpose. However, the Nevanlinna characteristic function was designed for just such purposes and indeed works in this case.

Once the theorem on exponentials is established, the two main theorems will follow from some linear algebra and combinatorial fiddling. $\$ 1$ will do this for 
Theorem 2, where it is simplest. Then, lest the geometry be camouflaged by equations, $\$ 2$ will indicate why the dimension drops so surprisingly in the general position theorem (Theorem 1 ) by looking at a specific case. $\$ 3$ gives the reduction of Theorem 1 to the theorem on exponentials. $\$ 4$ delineates the manner in which the bound $[n / k]$ of Theorem 1 is sharp through a construction more or less reversing that theorem's proof.

The next two sections contain the analysis. $\$ 5$ lists the properties of the Nevanlinna characteristic function we will need. $\$ 6$ establishes the theorem on linear relations among exponentials of holomorphic functions as a corollary of the following one variable theorem:

Theorem. Let $g_{i}: \mathbf{C} \rightarrow \mathbf{C}$ be bolomorphic functions, $b_{i}: \mathbf{C} \rightarrow \mathbf{P}_{1}$ meromorpbic functions, $i=1, \cdots, N$, satisfying

(1) $b_{1} \exp \left(g_{1}\right)+\cdots+b_{N} \exp \left(g_{N}\right) \equiv 1$,

(2) $T\left(b_{i}, r\right)$ is o $\left(\sum_{j=1}^{N} T\left(\exp \left(g_{j}\right), r\right)\right)$ for all $i=1, \ldots, N$, where $T$ is the Nevanlinna characteristic function and little o bas its usual meaning except that we exclude as values of $r$ a set of finite total measure in $\mathbf{R}^{+}$.

Then some proper linear combination of the terms $b_{i} \exp \left(g_{i}\right)$ is constant.

Out of the considerations of $\$ 6$ comes a result on algebraic independence of exponentials of meromorphic functions:

Theorem. Let $f_{1}, \cdots, f_{N}$ be bolomorpbic functions on $\mathrm{C}^{m}$ such that no nontrivial Z-linear combination of them is constant. Then $\exp \left(f_{1}\right), \ldots, \exp \left(f_{N}\right)$ are algebraically independent functions over $\mathbf{C}$.

$\S 7$ incorporates a few loose ends and suggests some improvements in the theorems.

During the last stages of preparation of this paper, news arrived of the existence of a paper of J. Dufresnoy from 1944 [4]. His work, although restricted to the case when the domain is one dimensional, has some overlap with mine. In particular, Corollary 2 of Theorem 1 should certainly be attributed to him. Using Dufresnoy's normal families methods, H. Fujimoto has independently found some of the results in this paper.

1. Proof of Theorem 2. We will assume the following lemma on exponentials of holomorphic functions, whose proof is found in a later section:

Theorem. Let $g_{i}: \mathrm{C}^{m} \rightarrow \mathrm{C}$ be bolomorphic functions, $i=0, \ldots, n$, satisfying identically

$$
\exp \left(g_{0}\left(z_{1}, \cdots, z_{m}\right)\right)+\cdots+\exp \left(g_{n}\left(z_{1}, \cdots, z_{m}\right)\right)=0
$$

Then for some distinct $i, j, g_{i}\left(z_{1}, \ldots, z_{m}\right)-g_{j}\left(z_{1}, \ldots, z_{m}\right)$ is identically constant. 
Recall the statement of Theorem 2:

Theorem 2. A holomorphic map $f: \mathbf{C}^{m} \rightarrow \mathbf{P}_{n}$ that omits any distinct $n+2$ byperplanes bas image lying in a proper projective linear subspace.

Proof. Let the hyperplanes be, in homogeneous coordinates,

$$
\left\{\sum_{i=0}^{n} a_{i t} z_{i}=0\right\}, \quad t=1, \cdots, n+2 .
$$

Writing $f$ as $\left(f_{0}, \cdots, f_{n}\right)$, if $f$ is to miss these hyperplanes, then $\sum_{i=0}^{n} a_{i t} f_{i}$ is never zero, nor can it have a pole since the $f_{i}$ have none; we can conclude it is the exponential exp $\left(g_{t}\right)$ of a holomorphic function $g_{t}: \mathbf{C}^{m} \rightarrow \mathrm{C}$. Doing this for each $t=1, \cdots, n+2$ yields a set of equations:

$$
\sum_{i=0}^{n} a_{i t} f_{i}=\exp \left(g_{t}\right), \quad t=1, \cdots, n+2 .
$$

Considering the $n+2$ vectors $a_{t}=\left(a_{0 t}, \cdots, a_{n t}\right)$ in $\mathbf{C}^{n+1}$, we note there must be at least one linear dependence among them, say $\sum_{t=1}^{n+2} c_{t} a_{t}=0$. Then

$$
\sum_{t=1}^{n+2} c_{t} \exp \left(g_{t}\right) \equiv 0 .
$$

By choosing logarithms for the nonzero $c_{t}$, we are in possession of a relation, if $S=\left\{t c_{t} \neq 0\right\}$,

$$
\sum_{t \in S} \exp \left(g_{t}+\log c_{t}\right) \equiv 0
$$

that falls under the umbrella of the theorem on exponentials that began this section. We are free to conclude, for some $s \neq t$, that $g_{s}-g_{t}$ is a constant $c$. So $\exp \left(g_{s}\right)=$ $\exp (c) \exp \left(g_{t}\right)$.

To return to the system of equations by which the $g$ 's were defined, we have

$$
\sum_{i=0}^{n} a_{i s} f_{i}=\exp \left(g_{s}\right), \quad \sum_{i=0}^{n} a_{i t} f_{i}=\exp \left(g_{t}\right) .
$$

Subtracting $\exp (c)$ times the second equation from the first produces

$$
\sum_{i=0}^{n}\left(a_{i s}-\exp (c) a_{i t}\right) f_{i} \equiv 0
$$

which is the linear relation among the $f_{i}$ the theorem requires. It is a nontrivial relation, for otherwise $a_{s}=\exp (c) a_{t}$, which would imply $a_{s}$ and $a_{t}$ determine the same hyperplane, contrary to hypothesis. Q.E.D.

2. Geometric interlude-Theorem 1 for $\mathbf{P}_{3}$. This section, it is hoped, will make the geometric content of Theorem 1 clearer and render its conclusion more 
plausible. We will take as our starting point Theorem 2 and see how, when the hyperplanes in question are in general position, the dimension of the image may be pared down still further. The general case will be treated formally in the next section-here we will spotlight the first interesting case, $\mathbf{P}_{3}$. Thus Theorem 1 becomes

Theorem 1 for $\mathbf{P}_{3}$. A bolomorphic map $f: \mathbf{C}^{m} \rightarrow \mathbf{P}_{3}$ that misses 5 byperplanes in general position goes into a complex line; a map that misses 7 byperplanes in general position is constant.

Proof from Theorem 2. Let $H_{1}, \cdots, H_{5}$ be 5 hyperplanes in general position in $\mathbf{P}_{3}$. By Theorem 2, we know the image of $f$ lies in a 2-plane in $\mathbf{P}_{3}$. But in order to avoid a further dose of Theorem 2, this 2-plane must intersect the 5 hyperplanes in only three distinct lines. This can happen only if the two-plane goes through two of the lines of intersection of pairs of the 5 hyperplanes (by general position, no three of the hyperplanes share a commonline). Further, the four hyperplanes involved in the intersections must be distinct, for if the lines were $H_{1} \cap$ $\mathrm{H}_{2}$ and $\mathrm{H}_{2} \cap \mathrm{H}_{3}$, then the 2-plane would have two lines in common with $\mathrm{H}_{2}$ and thus be $\mathrm{H}_{2}$, which we exclude, as $\mathrm{H}_{2}$ is omitted by the image. But the lines $H_{1} \cap H_{2}$ and $H_{3} \cap H_{4}$ contain no point in common, so there is no 2-plane passing through both to be found. We are led to conclude that even the best engineered of 2-planes intersects five hyperplanes in general position in $\mathbf{P}_{3}$ in at least four distinct lines. Theorem 2 compels the image of $f$ to lie in a line.

In fact, unless $f$ is constant, image $f$ lies in one of a very special class of lines, namely those lines joining one of the points of intersection of three hyperplanes among the five to a point on the line of intersection of the other two. It is worth noting that through a fixed point not lying on one of the 5 omitted hyperplanes, there are only a finite number of such lines. Hence, if we knew Theorem 2 only when the domain is $\mathbf{C}^{1}$, we could still conclude Theorem 1 for $\mathbf{P}_{3}$ for maps on any $\mathrm{C}^{m}$ by using the one-dimensional theorem on the lines through the origin in $\mathrm{C}^{m}$, because of the finite number of permissible image lines through $f(0)$. This argument, which is not particular to $\mathbf{P}_{3}$, illuminates the reasons that the transition from domain $\mathbf{C}^{1}$ to an arbitrary $\mathbf{C}^{m}$ is difficult when attention is confined to hyperplanes in general position and becomes rather natural when a less restricted class of hyperplanes is considered.

If six hyperplanes in general position in $\mathbf{P}_{3}$ are omitted, the permissible dimension of the image is not further reduced, as a line through two triple points, say $\mathrm{H}_{1} \cap \mathrm{H}_{2} \cap \mathrm{H}_{3}$ and $\mathrm{H}_{4} \cap \mathrm{H}_{5} \cap \mathrm{H}_{6}$, intersects the collection of six hyperplanes in just two distinct points. There is no slack in this case so that the addition of a seventh hyperplane in general position with respect to the others forces the line to intersect the system of hyperplanes in three distinct points. By the 
classical Picard theorem, $f$ is then constant.

3. Proof of Theorem 1. The only analytic fact needed in this section is the theorem on linear relations among exponentials; the rest is linear algebra. We are to prove

Theorem 1. Let $f: \mathbf{C}^{m} \rightarrow \mathbf{P}_{n}$ be a bolomorphic map that omits $n+k$ byperplanes in general position, $k \geq 1$. Then the image of $f$ is contained in a projective linear subspace of dimension $\leq[n / k]$, where the brackets mean greatest integer.

Proof. Let $\left(f_{0}, \cdots, f_{n}\right)$ be homogeneous coordinates of $f: \mathbf{C}^{m} \rightarrow \mathbf{P}_{n}$. Let the $n+k$ hyperplanes in general position be $\left\{\sum_{i=0}^{n} a_{i t} z_{i}=0\right\}$ where $t=1, \cdots, n+k$. If $f$ is to miss these hyperplanes, then $\sum_{i=0}^{n} a_{i t} f_{i}$ is never zero or infinite; the expression is therefore an exponential $\exp \left(g_{t}\right)$ where $g_{t}: \mathbf{C}^{m} \rightarrow \mathbf{C}$ are holomorphic. This yields $n+k$ equations:

$$
\sum_{i=0}^{n} a_{i t} f_{i}=\exp \left(g_{t}\right), \quad t=1, \cdots, n+k
$$

We group the $g_{t}$ into collections so that $g_{t}$ and $g_{u}$ are in the same cluster iff they differ by a constant. Assume there are $s$ such clusters, each one as large as possible.

The equations are now relabeled so that $g_{1}, \ldots, g_{s}$ are representatives, one from each cluster. Equations are multiplied by constants so that the right-hand side is always one of the functions $\exp \left(g_{r}\right), r=1, \cdots, s$. The notation can be altered so that $I_{1}, \ldots, I_{s}$ partition the set $\{1, \ldots, n+k\}$ with the property that

$$
\sum_{i=0}^{n} a_{i t} f_{i}=\exp \left(g_{r}\right) \text { iff } t \in I_{r} .
$$

The following properties of hyperplanes in general position in $\mathbf{P}_{n}$ are recalled:

(1) Any $n+1$ of the vectors $a_{t}=\left(a_{0 t}, \cdots, a_{n t}\right)$ are linearly independent.

(2) Any $n+2$ of the vectors $a_{t}$ satisfy a linear relation none of whose coefficients is zero (or else there would be a dependence between $n+1$ of the vectors).

Now, if the complement of any $I_{r}$ in the set $\{1, \ldots, n+k\}$ contained $n+1$ or more elements, it would become possible to pick a subset $S$ of $\{1, \cdots, n+k\}$ of $n+2$ indices so that $S \cap I_{r}$ consisted of precisely one element. The linear relation among the vectors of $S$ would induce a linear relation among the $\exp \left(g_{t}\right)$, and after we regroup the terms coming from the same cluster, there would still be a nontrivial linear equation among the $\exp \left(g_{t}\right)$. The relation is nontrivial because the coefficient of $\exp \left(g_{r}\right)$ is nonzero, which is a consequence of the fact $S \cap I_{r}$ contains a unique element. Such an equation would contradict the theorem on linear 
relations of exponentials, for after the terms are collected in clusters, no $g_{t}$ and $g_{u}$ differ by a constant for $t \neq u$. Contradiction, so the complement of any $I_{r}$ in the set $\{1, \cdots, n+k\}$ contains at most $n$ elements. Therefore, $I_{r}$ contains at least $k$ equations for all $r=1, \ldots, s$. In consequence, the number of clusters $s$ is at most $[(n+k) / k]$ where the brackets mean greatest integer.

All that remains is to interpret $s$. To do this, pick any subset $T$ of $n+1$ equations of $\{1, \cdots, n+k\}$. Write $T=\bigcup_{r} T_{r}$, where $T_{r}=T \cap I_{r}$. Then the \#(Tr) equations in each $T_{r}$ yield $\#\left(T_{r}\right)-1$ linearly independent linear equations involving only the $f_{i}$ and none of the $\exp \left(g_{t}\right)$, for we can subtract adjacent equations in the same cluster to cancel out the exponentials with end product $\sum_{i=0}^{n}\left(a_{i, t}-a_{i, t+1}\right) f_{i}=0$ where $t, t+1 \in T_{r}$. Doing this for each of the $s$ clusters yields $\#(T)-s=n+1-s$ linearly independent equations among the $f_{i}$. (The reason the equations are independent follows from the fact that the original $n+1$ equations we chose to form $T$ were linearly independent, which was a consequence of general position.) So the image of $f$ is contained in a linear subspace of $\mathbf{P}_{n}$ of codimension $n+1-s$, hence of dimension $s-1$.

We already know $s \leq[(n+k) / k]$, so $s-1 \leq[n / k]$. So the image of $f$ is contained in a linear subspace of dimension $\leq[n / k]$. Q.E.D.

4. Sharpness of Theorem 1. Although the dimension $[n / k]$ of Theorem 1 may appear to be a consequence of the method of proof rather than a geometrically significant number, it is indeed the sharpest possible bound and is always attained for any given $n+k$ hyperplanes in general position. In fact, $[n / k]$ can always be realized as the actual dimension of the image of a nondegenerate map from $\mathbf{C}^{[n / k]}$ to $\mathbf{P}_{n}$ omitting the hyperplanes, and not merely as the dimension of the minimal linear subspace of $\mathbf{P}_{n}$ containing the image.

To see this, let $s=[n / k]+1$. Let the $n+k$ hyperplanes in general position be represented by vectors in homogeneous coordinates $a_{t}=\left(a_{0 t}, \cdots, a_{n t}\right)$. Let $l_{1}, \cdots, I_{s}$ be a partition of $\{1, \cdots, n+k\}$ so that each $I_{m}$ contains $k$ or more elements.

Select holomorphic functions $g_{m}: \mathrm{C}^{[n / k]} \rightarrow \mathbf{C}, m=1, \ldots, s$, so the map $\left(\exp g_{1}, \ldots, \exp g_{s}\right): \mathbf{C}^{[n / k]} \rightarrow \mathbf{P}_{[n / k]}$ is nondegenerate (such maps abound-for example $\left.g_{1}=z_{1}, \cdots, g_{s-1}=z_{s-1}, g_{s}=1\right)$. We seek a map $f=\left(f_{0}, \ldots, f_{n}\right)$ : $\mathbf{C}^{[n / k]} \rightarrow \mathbf{P}_{n}$ so that $\sum_{i=0}^{n} a_{i t} f_{i}=d_{t} \exp \left(g_{m}\right)$ where $t \in I_{m}$ and $d_{t} \neq 0$ for all $t=1, \cdots, n+k$. If we can find nonzero $d_{t}$ so that these $n+k$ equations reduce to only $n+1$ equations, independent and compatible, then $f$ will be a map with the properties we seek.

Let $r_{b}=\left(r_{1, b}, \cdots, r_{n+2, b}\right)$ be a basis for the linear relations among the $n+k$ hyperplanes, $b=1, \ldots, k-1$. We want to pick $d_{t}, t=1, \cdots, n+k$, to satisfy 
the system of equations:

$$
\sum_{t \in I_{m}} r_{t b} d_{t}=0 \text { for all } m=1, \cdots, s \text { and } b=1, \cdots, k-1 .
$$

This is a system of $(k-1) s$ equations that breaks up into the $s$ subsystems we get by keeping $m$ fixed. Each subsystem consists of $k-1$ equations in $\#\left(I_{m}\right) \geq$ $k$ unknowns. Finding a nontrivial solution is no problem, but we want one with all $d_{t} \neq 0$. It would be impossible to find such a solution only if some linear combination of the equations of the subsystem involved only one of the variables. The hypothesis of general position of the hyperplanes implies that any relation involves at least $n+2$ of them. Hence any linear combination of the $r_{b}$ must have at least $n+2$ nonzero entries. As $\#\left(I_{m}\right) \geq k$, at least 2 of the nonzero entries must lie in $I_{m}$. Therefore the situation which would prevent a solution of the subsystem with all $d_{t} \neq 0, t \in I_{m}$, is ruled out by the assumption of general position. Because the $s$ subsystems share no variables in common, we can put the solutions together to obtain a solution $d_{t}, t=1, \cdots, n+k$, of all the equations with no $d_{t}$ being zero.

The equations were set up so that the $n+k$ equations $\sum_{i=0}^{n} a_{i t} f_{i}=d_{t} \exp \left(g_{m}\right)$, $t=1, \cdots, n+k$, reduce to (say) the first $n+1$ of them. Letting $A$ be the $(n+1) \times(n+1)$ matrix $\left(a_{i t}\right), 0 \leq i \leq n, 1 \leq t \leq n+1$, nonsingular by general position, then $A(f)=b$, where $b$ is the column vector $\left(d_{t} \exp \left(g_{m}\right)\right), t=1, \cdots, n+1$. Therefore $f=A^{-1}(b)$. Having picked the $g_{m}$, we take this to be the definition of $f$. It follows from the construction that $f: \mathrm{C}^{[n / k]} \rightarrow \mathrm{P}_{n}$ goes nondegenerately to its image in an $[n / k]$ dimensional linear subspace of $\mathbf{P}_{n}$ and omits the $n+k$ hyperplanes we started with.

As an example of the class of maps constructed, we remark that for $k=n$ we get the nonconstant maps of the complex line to $\mathbf{P}_{n}$ minus $2 n$ hyperplanes in general position whose existence was pointed out by P. J. Kiernan [5]. We take, as $s=2, I_{1}=$ any subset of $n$ elements of $\{1, \ldots, 2 n\}, I_{2}$ the $n$ remaining elements. Then the line in which the image lies is the one passing through the point of intersection of the hyperplanes indexed by $I_{1}$ and the corresponding point for $I_{2}$. Any nonconstant map from $\mathrm{C}$ to this line omitting those two points gives an example.

5. The Nevanlinna characteristic function. Among the many beautiful techniques that enliven complex analysis, only a few can rival the elegance, power, and simplicity of the Nevanlinna characteristic function. Even its most elementary properties suffice for the purposes we have in mind, so it is these we will briefly recapitulate. An ideal reference is [6].

Let $f: \mathbf{C} \rightarrow \mathbf{P}_{1}$ be a meromorphic function of one variable (much of what follows carries over to several variables). Consider, for $a \in \mathbf{P}_{1}$, 


$$
\begin{aligned}
n(r, a) & =\#\left\{z \in f^{-1}(a)|| z \mid \leq r\right\} \text { counting multiplicities, } \\
N(r, a) & =\int_{0}^{r} \frac{n(t, a)-n(0, a)}{t} d t+n(0, a) \log r \\
m(r, a) & = \begin{cases}\int|z|=\log ^{+}\left|\frac{1}{f(z)-a}\right| d \theta & \text { if } a \neq \infty \\
\int_{|z|=r} \log ^{+}|f(z)| d \theta & \text { if } a=\infty\end{cases}
\end{aligned}
$$

where $\log ^{+} x=\max (\log x, 0)$. The function $N(r, a)$ is called the integrated counting function and is continuous in $r$. The function $m(r, a)$ is called the proximity function because it measures how closely $f$ approaches the value $a$ on the circle of radius $r$. If we let

$$
T(r)=N(r, a)+m(r, a),
$$

then it is a consequence of Jensen's theorem that this expression is independent of $a$ in the sense that the difference of any two such is bounded. This is the First Main Theorem of Nevanlinna theory and it allows us to define the Nevanlinna characteristic function $T(r)$ by the above equation.

We can define $T(r)$ a different way. Let $\omega$ be the Fubini-Study metric on $\mathbf{P}_{1}$, normalized so that $\int_{\mathrm{P}_{1}} \omega=1$. If $B(t)$ is the disc of radius $t$, then

$$
T(r)=\int_{0}^{r} \int_{B(t)} f^{*}(\omega) \frac{d t}{t} .
$$

Thus $T(r)$ is the logarithmically averaged volume of the images under $f$ of discs of radius $\leq r$, where points in the image repeatedly assumed by $f$ are counted each time they are hit. This representation of the characteristic function shows that

(1) $T(r)$ is a continuous, increasing function of $r$,

(2) $\lim _{r \rightarrow \infty} T(r)=\infty$ unless $f$ is constant, in which case it remains bounded.

Some other fairly easy properties of the Nevanlinna characteristic function that will be used later are

(3) $T(f+g, r) \leq T(f, r)+T(g, r)+\log 2$,

(4) $T(f g, r) \leq T(f, r)+T(g, r)$,

(5) $T(1 / f, r)=T(f, r)+$ constant.

Two less elementary properties will be needed, relating to the characteristic functions of derivatives and exponentials of a function $f$. In order to state these, two conventions of notation to be used in this section and the next will assist us:

Convention. $A(r)=o(B(r))$ will mean $B(r) \not \equiv 0$ and $\lim _{r \rightarrow \infty} A(r) / B(r)=0$ after a set of intervals of $\mathbf{R}^{+}$of finite total measure are excluded as values of $r$. Likewise, $A(r)=O(B(r))$ will mean that there exists a constant $c$ so that $A(r) \leq c B(r)$ after excluding a set of intervals as above. Then

(6) $T\left(f^{\prime}, r\right)=O(T(f, r))$ where $f^{\prime}$ is the derivative of $f$, 
(7) for $f$ a nonconstant holomorphic function, $T(f, r)=o\left(T\left(e^{f}, r\right)\right)$.

The exceptional intervals which have intruded here plague many of the proofs in Nevanlinna theory, but fortunately they can most often be quarantined from appearing in the statements of the theorems. Their presence is due to a persistently useful lemma of Emile Borel that exacts such exceptional intervals as the price of its conclusion. Because the union of a finite number of sets of finite total measure is likewise of finite total measure, the modified $O$ and $O$ symbols retain the congenial properties of their orthodox counterparts, and indeed exceptional intervals will necessitate no modification of the method of proof.

6. Linear relations of exponentials of entire functions. The analytic sinews of the main theorems will be our concern in this section. The theorem on exponentials used to prove Theorems 1 and 2 will itself be proved. The results in the theory of entire functions of one complex variable to which that theorem will reduce have been known since 1897, and the method of proof goes back to Nevanlinna. Since then, their approach has fallen into the penumbra of dimly remembered mathematics. For this reason I will give a complete proof, albeit somewhat different from the originals. In the course of it, a result in one variable will surface similar to E. Borel's classical improvement of Picard's theorem.

The theorem on exponentials we are aiming for is

Theorem. Let $g_{i}: \mathrm{C}^{m} \rightarrow \mathrm{C}$ be bolomorpbic functions, $i=1, \cdots, n$, satisfying identically

$$
\exp \left(g_{1}\right)+\cdots+\exp \left(g_{n}\right)=0 .
$$

Then for some distinct $i, j$, the function $g_{i}-g_{j}$ is identically constant.

Proof. The first step is a reduction to the case $m=1$, where the domain of the functions is a complex line. Assuming the result in this case, consider the restriction of the $g_{i}: \mathrm{C}^{m} \rightarrow \mathrm{C}$ to a line $\xi$ through the origin. On $\xi$, the equation of exponentials for the restricted functions $\left.g_{i}\right|_{\xi}$ still holds; hence for some distinct $i$ and $j, g_{i}\left|\xi-g_{j}\right|_{\xi}$ is the constant $g_{i}(0)-g_{j}(0)$. Let $B_{i j}=\left\{\xi \in \mathbf{P}_{m-1}\right.$ : $\left.g_{i}\right|_{\xi}-\left.g_{j}\right|_{\xi}$ is constant $\}$. Because the theorem is assumed to hold for every line, $\bigcup_{i \neq j} B_{i j}$ is all of $\mathbf{P}_{m-1}$. By the category theorem, as the $B_{i j}$ are closed, one of the $B_{i j}$ contains an open set. But now the identity theorem takes over, for an open set in the set of lines through the origin sweeps out a region in $\mathrm{C}^{m}$ containing an open set on which $g_{i}-g_{j}$ is the constant $g_{i}(0)-g_{j}(0)$. Thus the constant and the function are identical on all of $\mathrm{C}^{m}$. This completes the reduction to one variable.

The inductive process sketched in the Introduction-dividing through by one term, differentiating, dividing through again getting an ever shorter equation and eventually a contradiction-may now be applied. Before tackling the real thing, it 
is instructive to work out the case where the exponentials in question are entire of finite order, i.e. exponentials of polynomials. So assume

$$
\exp \left(P_{1}(z)\right)+\cdots+\exp \left(P_{N}(z)\right) \equiv 0 .
$$

Dividing through by the last term and differentiating gives

$$
\sum_{i=1}^{N-1}\left(P_{i}^{\prime}(z)-P_{N}^{\prime}(z)\right) \exp \left(P_{i}(z)-P_{N}(z)\right) \equiv 0 .
$$

The coefficients can vanish only when $P_{i}(z)-P_{N}(z)$ is constant, so the new equation is nontrivial. Continuing the process, at the $(N+1-k)$ th stage we get

$$
\sum_{i=1}^{k-1} R_{i}(z) \exp \left(P_{i}(z)-P_{k}(z)\right)=1
$$

where the $R_{i}(z)$ are nonzero rational functions. When we differentiate this equation, if we lose any terms it means some $R_{i}(z) \exp \left(P_{i}(z)-P_{k}(z)\right)$ is constant. This is impossible unless $P_{i}(z)-P_{k}(z)$ is constant, since the exponential of a nonconstant polynomial is a transcendental function. As the proof is done if any $P_{i}(z)-P_{k}(z)$ is constant, we may as well assume it is not and continue the induction, which leads inexorably to the conclusion we have just tried to reject. For at the $(N-1)$ th stage, we get

$$
R_{1}(z) \exp \left(P_{1}(z)-P_{2}(z)\right)=1 \text {. }
$$

This can only happen if $P_{1}(z)-P_{2}(z)$ is constant. Thus somewhere along the line, a $P_{i}(z)-P_{j}(z)$ is constant, $i \neq j$.

The same proof will work to show that the conclusion holds if we assume the coefficients of our equation of exponentials of polynomials to be rational functions instead of constants, so

$$
\sum_{i=0}^{N} R_{i}(z) \exp \left(P_{i}(z)\right)=0
$$

will imply $P_{i}-P_{j}$ is constant for some distinct $i, j$. It is this stronger result that will be generalized. The difficulty is how to generalize the fact that sums, products, derivatives, and quotients of rational functions remain rational functions, and the fact that the exponential of a rational function can be a rational function only if it is constant. If we take equations of exponentials whose coefficients have Nevanlinna characteristic functions that are "little $o$ " of the characteristic functions of the exponentials themselves, then the properties of the characteristic function listed in the preceding section will perform the role of the needed features of the rational functions.

To be precise, what can be shown is 
Theorem. Let $g_{i}: \mathbf{C} \rightarrow \mathbf{C}$ be entire functions, $b_{i}: \mathbf{C} \rightarrow \mathbf{P}_{1}$ meromorpbic functions, $i=1, \cdots, N$, satisfying

(1) $b_{1} \exp \left(g_{1}\right)+\cdots+b_{N} \exp \left(g_{N}\right) \equiv 1$,

(2) $T\left(b_{i}, r\right)$ is o $\left(\sum_{j=1}^{N} T\left(\exp \left(g_{j}\right), r\right)\right)$ for all $i=1, \cdots, N$, where $T$ denotes the Nevanlinna characteristic function and little o has its usual meaning except that we may exclude as values of $r$ a set of intervals of $\mathbf{R}^{+}$of finite total measure (as in $\$ 5$ ).

Then some proper nontrivial linear combination of the terms $b_{i} \exp \left(g_{i}\right)$ is constant.

Remark 1. The relation of the conclusion cannot necessarily be shortened further, for the hypotheses are not hereditary. Consider for example $b_{1}=\exp \left(z^{3}\right)$, $g_{1}=z^{4}, b_{2}=-1, g_{2}=z^{4}+z^{3}, b_{3}=\left(e^{z}+1\right) / e^{z}, g_{3}=z, b_{4}=-1, g_{4}=z$, which satisfy the theorem's hypotheses. Then in fact, $b_{3} \exp \left(g_{3}\right)+b_{4} \exp \left(g_{4}\right)=1$, but while $T\left(b_{3}\right)$ is $o\left(\sum_{i=1}^{4} T\left(\exp \left(g_{i}\right), r\right)\right)$, it is not little of $T\left(\exp \left(g_{3}\right), r\right)+$ $T\left(\exp \left(g_{4}\right), r\right)$.

Remark 2. There is, however, an important subcase for which the hypotheses are hereditary-the theorem on linear relations among exponentials that was grist for the combinatorial mills of the first few sections. The verification is easy. Assume relation $\sum_{i=1}^{N} \exp \left(g_{i}\right) \equiv 0$. This may be rephrased as $\sum_{i=1}^{N-1} \exp \left(g_{i}-g_{N}\right) \equiv$ - 1. The present theorem now applies, the $b_{i}$ being constants, so the $T\left(b_{i}\right)$ are constant and thus $o\left(T\left(\exp \left(g_{i}-g_{N}\right)\right)\right)$ unless $g_{i}-g_{N}$ is constant, in which case we would be done. So some proper linear combination $\Sigma_{i \in S} \exp \left(g_{i}-g_{N}\right)$ is constant, as is the sum over the complement of $S$. Since the two constants add up to -1 , they cannot both be zero. The theorem may be used again on whichever of the two linear combinations is nonzero until we reap the conclusion that, for some $i$ different from $N, \exp \left(g_{i}-g_{N}\right)$ is constant and nonzero. This gives a conclusion superficially stronger than the theorem we are trying to prove in that it says, for every $i$, there exists a $j \neq i$ with $g_{i}-g_{j}$ constant. In fact, a simple induction is all that separates the strong form of the theorem from the weak. The "clusters" of the proof of Theorem 1 are equivalent to this strong form.

Proof of the theorem. Another induction, again on the length of the relation, will be used. The theorem is clear for $N=1$, as $b_{1} \exp \left(g_{1}\right) \equiv 1$ implies $T\left(b_{1}, r\right)=$ $T\left(\exp \left(g_{1}\right), r\right)$, but then $T\left(b_{1}, r\right)$ is assumed to be $o\left(T\left(b_{1}, r\right)\right)$, which cannot be, exceptional intervals notwithstanding.

Assume the theorem true for lengths shorter than $N$, and let $\sum_{i=1}^{N} b_{i} \exp \left(g_{i}\right)=$ 1 , with hypotheses as above and $N>1$. Differentiating, we get $\sum_{i=1}^{N} x_{i} \exp \left(g_{i}\right)=$ 0 where $x_{i}=b_{i}^{\prime}+b_{i} g_{i}^{\prime}$. We note, as we are working in one variable, that $x_{i} \equiv 0$ iff $b_{i} \exp \left(g_{i}\right)$ is constant. But this would be a constant proper linear combination, and thus the requirements of the conclusion would be met, so we may assume this route 
of escape is closed off and $x_{i}$ is not identically zero. Therefore we may divide by the last term and get $-\sum_{i=1}^{N-1}\left(x_{i} / x_{N}\right) \exp \left(g_{i}-g_{N}\right) \equiv 1$. This relation would prove amenable to an application of the inductive hypothesis provided $T\left(x_{i} / x_{N}\right)$ is $o\left(\sum_{j=1}^{N-1} T\left(\exp \left(g_{j}-g_{N}\right)\right)\right)$ for all $i=1, \cdots, N-1$. The properties of the characteristic function listed in the last section combine to give that $T\left(x_{i} / x_{N}\right)$ is $o\left(\sum_{j=1}^{N} T\left(\exp \left(g_{j}\right)\right)\right)$, but this is not quite good enough.

We are in for a pleasant surprise-and this is what makes the proof work-that after a brief bit of legerdemain, the estimate we have on $T\left(x_{i} / x_{N}\right)$ is good enough after all. It turns out that $\sum_{i=1}^{N} T\left(\exp \left(g_{i}\right)\right)$ is $O\left(\sum_{j=1}^{N-1} T\left(\exp \left(g_{j}-g_{N}\right)\right)\right)$, which is surprising since the latter sum contains fewer functions. To see this estimate, we rewrite the original equation as

Therefore,

$$
\exp \left(-g_{N}\right)=\sum_{i=1}^{N-1} b_{i} \exp \left(g_{i}-g_{N}\right)+b_{N} .
$$

$$
\begin{aligned}
T\left(\exp \left(g_{N}\right)\right) & =T\left(\exp \left(-g_{N}\right)\right) \\
& \leq \sum_{i=1}^{N} T\left(b_{i}\right)+\sum_{i=1}^{N-1} T\left(\exp \left(g_{i}-g_{N}\right)\right)+\text { constant } .
\end{aligned}
$$

Using the hypothesis on the $T\left(h_{i}\right)$, and noting that $T\left(\exp \left(g_{i}\right)\right) \leq T\left(\exp \left(g_{N}\right)\right)+$ $T\left(\exp \left(g_{i}-g_{N}\right)\right)$, we get

$$
\begin{aligned}
T\left(\exp \left(g_{N}\right)\right) \leq o & \left(T\left(\exp \left(g_{N}\right)\right)\right)+o\left(\sum_{i=1}^{N-1} T\left(\exp \left(g_{i}-g_{N}\right)\right)\right) \\
& +\sum_{i=1}^{N-1} T\left(\exp \left(g_{i}-g_{N}\right)\right)+\text { constant } .
\end{aligned}
$$

But the little o's can be incorporated into the dominant terms to give

$$
T\left(\exp \left(g_{N}\right)\right) \leq 2 \sum_{i=1}^{N-1} T\left(\exp \left(g_{i}-g_{N}\right)\right)+\text { constant }
$$

(except possibly for exceptional intervals).

Since $T\left(\exp \left(g_{i}\right)\right) \leq T\left(\exp \left(g_{N}\right)\right)+T\left(\exp \left(g_{i}-g_{N}\right)\right)$, we conclude $\sum_{i=1}^{N} T\left(\exp \left(g_{i}\right)\right)$ is $O\left(\sum_{i=1}^{N-1} T\left(\exp \left(g_{i}-g_{N}\right)\right)\right)$.

As we now know that all of the necessary hypotheses are verified for the shorter relation we constructed, the inductive hypothesis ensures that a proper linear combination of the shorter equation's terms is constant, i.e.

$$
\sum_{i \in S} a_{i}\left(x_{i} / x_{N}\right) \exp \left(g_{i}-g_{N}\right)=c, \quad S \varsubsetneqq\{1, \ldots, N-1\} .
$$


This may be rearranged as

$$
\sum_{i \in S} a_{i} x_{i} \exp \left(g_{i}\right)-c x_{N} \exp \left(g_{N}\right)=0, \quad a_{i} \text { 's and } c \text { constants. }
$$

Recalling that $\left(b_{i} \exp \left(g_{i}\right)\right)^{\prime}=x_{i} \exp \left(g_{i}\right)$, we may integrate and get

$$
\sum_{i \in S} a_{i} b_{i} \exp \left(g_{i}\right)-c b_{N} \exp \left(g_{N}\right)=d, \quad d \text { a constant. }
$$

But this is a constant proper linear combination of the terms of the original equation, so we are done. Q.E.D.

Although it is not really germane to the main topic of this paper, the following theorem drops out for free:

Theorem (algebraic independence of exponentials of holomorphic functions). Let $f_{1}, \cdots, f_{N}$ be bolomorpbic functions on $\mathbf{C}^{m}$ such that no nontrivial Z-linear combination of them is constant. Then $\exp \left(f_{1}\right), \ldots, \exp \left(f_{N}\right)$ are algebraically independent functions over $\mathrm{C}$.

Proof. A polynomial relation among the exponentials may be rewritten as

$$
\sum_{j=1}^{M} c_{j} \exp \left(\sum_{i=1}^{N} a_{i j} f_{i}\right)=0, \quad a_{i j} \in \mathbf{Z}, c_{j} \in \mathbf{C}^{*}, M \text { some integer. }
$$

By the theorem on linear relations of exponentials, this implies that, for some $j \neq k, \log c_{j}+\sum_{i=1}^{N} a_{i j} f_{i}=\log c_{k}+\sum_{i=1}^{N} a_{i k} f_{i}$, and hence $\sum_{i=1}^{N}\left(a_{i j}-a_{i k}\right) f_{i}=$ $\log c_{k}-\log c_{j}$. This contradicts the hypothesis.

7. Further remarks. The case of maps omitting hyperplanes in other than general position is hardly settled by Theorem 2. An instance of the strange things that can happen is this:

Proposition. A bolomorphic map $F: \mathbf{C}^{m} \rightarrow \mathbf{P}_{n}$ that omits three byperplanes intersecting in a linear subspace of codimension 2 bas image lying in a byperplane.

This proposition may easily be proved by my methods, and in fact reduces to the classical Picard theorem. James Carlson has shown that a map omitting three elements of a linear pencil of divisors in $\mathbf{P}_{n}$ must have image lying in one element of the pencil, since a map is induced to the $P_{1}$ parameterizing the pencil and missing three points, so that Picard's theorem applies.

The method and point of view developed in this paper clearly allows one to determine in a finite number of steps for any set of hyperplanes a sharp bound for the dimension of linear spaces containing the images of maps into $\mathbf{P}_{n}$ omitting those hyperplanes. It would be nice to have something better-a general formula for this bound based on the intersection properties of the set of hyperplanes. Once the right formula is conjectured it should follow by the methods developed here. 
Theorems 1 and 2 might be slightly improved by replacing the hypothesis that the hyperplanes are omitted by the weaker assumption that the hypersurfaces each have Nevanlinna defect 1 in the sense of the classical theory of holomorphic curves. This should follow from the theorem we used to prove the theorem on linear relations of exponentials of holomorphic functions.

A more interesting direction to pursue is the case of maps omitting divisors on $\mathbf{P}_{n}$ of degree $n+2$ or greater. Considerable progress on this problem is made in James Carlson's thesis [2] and his recent joint work with Phillip Griffiths.

Another class of problems is to consider maps defined only locally, on a ball or polydisc, and obtain generalizations of the Schottky-Landau theorem and results on hyperbolicity.

Acknowledgments. It is a pleasure to acknowledge the generous assistance of Phillip Griffiths. Of great help, too, have been many conversations with James Carlson and $\mathrm{H}$. Wu.

\section{BIBLIOGRAPHY}

1. E. Borel, Sur les zéros des fonctions entières, Acta Math. 20 (1887), 357-396.

2. James Carlson, Some degeneracy theorems for entire functions with values in an algebraic variety, Thesis, Princeton University, Princeton, N. J., 1971.

3. S. S. Chern, Proceedings International Congress of Mathematicians (Nice, 1970).

4. J. Dufresnoy, Théorie nouvelle des. famillies complexes normales. Applications à l'étude des fonctions algébroides, Ann. Sci. École Norm. Sup. (3) 61 (1944), 1-44. MR 7.289.

5. Peter Kiernan, Hyperbolic submanifolds of complex projective space, Proc. Amer. Math. Soc. 22 (1969), 603-606. MR 39 \#7134.

6. R. Nevanlinna, Le théorème de Picard-Borel et la théorie des fonctions méromorphes, Gauthier-Villars, Paris, 1929.

7. $\mathrm{H} . \mathrm{Wu}$, The equidistribution theory of holomorphic curves, Princeton Univ. Press, Princeton, N. J., 1970.

8. $\longrightarrow$ An n-dimensional extension of Picard's theorem, Bull. Amer. Math. Soc. 75 (1969), 1357-1361. MR $40 \# 7482$.

DEPARTMENT OF MATHEMATICS, PRINCETON UNIVERSITY, PRINCETON, NEW JERSEY 08540

Current address: Department of Mathematics, University of California, Berkeley, California 94720 